\title{
EL FUTURO DE LA FILOSOFÍA DEL DERECHO*
}

\author{
Luigi Ferrajoli \\ Universidad de Roma III
}

\begin{abstract}
RESUMEN. Esta es la conferencia pronunciada por Luigi FERRAJol en el I Congreso de Filosofía del Derecho para el Mundo Latino (i-Latina). En ella, el autor identifica las dos afinidades que, en su opinión, justifican hablar de una iusfilosofía jurídica del Mundo Latino: por un lado, la confianza en la razón como factor de civilización del derecho y de limitación de la arbitrariedad, así como el vinculo estrecho entre reflexión iusfilosófica y práctica jurídica; y, por otro, el que las elaboraciones teóricas de la Región reflejan características estructurales presentes en sus democracias constitucionales que no aparecen con igual rotundidad en los sistemas del common law. A partir de ahí, FERRAJOLI caracteriza, en clave positivista y antirealista, las que deberían ser las principales tareas de la filosofía del derecho del futuro.
\end{abstract}

Palabras clave: Anomia, derechos sociales, FerRAJolı, globalización, i-Latina, Mundo Latino, poderes salvajes, positivismo, realismo.

\section{The future of Philosophy of Law}

ABSTRACT. This is FerRajolis' lecture at the I Congreso de Filosofía del Derecho para el Mundo Latino (i-Latina). The author identifies the two similarities that -in his opinion- justify speaking of a legal philosophy for the Latin World: one is the share trust on the role of reason as civilising factor since it limits arbitrariness and keeps Law restrained, and as the bond between iusphilosophical reflection and legal practice. Another is the fact that theoretical elaborations in the Region reflect the very structural characteristics in their constitutional democracies, which happen not to be equally present in the common law systems. Assuming this framing, FERRAJOLI characterises, on the bases of positivism and antirealism, the principal tasks that legal philosophy should be undertaking hereafter.

Keywords: Anomie, social rights, FerRAJOLI, globalisation, i-Latina, Latin World, Unrestrained powers, positivism, realism.

* Fecha de recepción: 4 de junio de 2016. Fecha de aceptación: 21 de septiembre de 2016.

Traducción de Alí LOZADA. 


\section{PASADO, PRESENTE Y FUTURO DE LA FILOSOFÍA DEL DERECHO EN EL MUNDO LATINO}

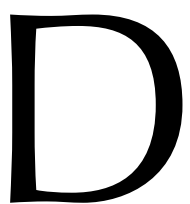

ebemos agradecer a Manuel ATIENZA por la iniciativa de este magnífico Congreso, que ha mostrado, en sus numerosas relatorías y en el intenso debate posterior a cada una de ellas, la pluralidad de ideas y enfoques que caracterizan a la filosofía del derecho en nuestro Mundo Latino, así como el clima cultural común ligado a nuestras tradiciones culturales compartidas y, más aún, a las similares características estructurales de nuestros ordenamientos. Añado que el debate en los últimos días ha demostrado que nuestras similitudes son mucho mayores que nuestras diferencias, y que la confrontación entre nosotros es un factor que asegura el enriquecimiento y desarrollo de nuestras disciplinas. Creo que, por encima de todo, hay dos afinidades que permiten hablar de una filosofía jurídica específica del Mundo Latino.

La primera afinidad, ya destacada por Manuel ATIENZA, consiste en la común confianza en la razón como factor de civilización del derecho y de limitación de la arbitrariedad, así como en la estrecha relación instituida entre reflexión iusfilosófica y práctica jurídica. Agrego que estas dos características de nuestras culturas filosófico-jurídicas se han afirmado, en casi todos nuestros países, con la finalización de los regímenes fascistas o autoritarios. Con el advenimiento de las democracias, primero en Italia, luego en España y después en América Latina, se produjo un giro en el pensamiento filosófico-jurídico, orientado al redescubrimiento de la razón y al desarrollo de disciplinas empíricas, analíticas y anti-metafísicas: la teoría general del derecho, la metodología de la ciencia jurídica, el análisis del lenguaje jurídico, la lógica jurídica, la teoría de la argumentación y de la interpretación y, en general, del razonamiento jurídico. Es extraordinaria la coincidencia de este cambio de enfoque filosófico con la finalización de los regímenes totalitarios o autoritarios. Obviamente, sería arbitrario cualquier juicio acerca de las valencias políticas de las distintas orientaciones filosóficas. Aludo solamente al nexo entre clima político y clima filosófico que bien advirtiera Hans KELSEN en un bellísimo artículo de 1932 titulado Defensa de la Democracia: «En los círculos teóricos del derecho público y en los sociólogos», escribió en vísperas del nacismo, «hoy en día es casi natural hablar de la democracia con palabras burlonas, mientras que está de moda saludar a la dictadura, directa o indirectamente, como al amanecer de una nueva era. Y este cambio de actitud "científica" va de la mano con un cambio en el frente filosófico; de la claridad del razonamiento empírico-crítico, desprestigiado como trivialidad, se vuelve a la oscuridad, considerada profundidad, de la metafísica, al culto a un irracionalismo nebuloso, es en esta atmósfera específica donde mejor han prosperado las más diversas formas de autocracia» (en H. KelSEN, Sociologia della democrazia, a cura di A. CARRINO, Napoli, Esi, 1994: 42-43).

Hay un segundo factor que, más allá de nuestras diferencias, es común a nuestra manera de concebir y practicar la filosofía del derecho y permite hablar de una específica filosofía del derecho del Mundo Latino: el hecho, ya mencionado, de que nuestros enfoques reflejan características estructurales de nuestras democracias constitucionales, que no se encuentran con la misma rotundidad en los sistemas del common law y 
que nuestra subordinación a la cultura jurídica anglosajona nos hace a veces olvidar. La primera de estas características es el principio iuspositivista de legalidad como norma de reconocimiento de todo el derecho existente. La segunda es la constitucionalización rígida de principios de la justicia como el principio de igualdad, los derechos de libertad y, sobre todo, los derechos sociales, como límites y vínculos impuestos a los poderes públicos. Es este constitucionalismo rígido la característica más relevante de nuestros ordenamientos, el que ha conferido a la reflexión sobre los derechos fundamentales y sus garantías una centralidad teórica que es, sin duda, uno de los aspectos específicos de nuestra filosofía jurídica.

Ahora, cabe preguntarse: ¿qué tipo de enseñanza filosófico-jurídica es requerida por estas dos propiedades estructurales de nuestras democracias de cara a la formación de los juristas y, ante todo, al desarrollo del interés por el derecho en nuestros estudiantes? Siempre he creído que el principal propósito de nuestra enseñanza de filosofía del derecho es infundir en los estudiantes un interés por el derecho capaz de vencer el tedio generado, sobre todo en el estudio de las disciplinas jurídicas más especializadas, por la sensación de estudiar una técnica burocrática de ejercicio del poder y de control social. Pues bien, el desarrollo en nuestros estudiantes del interés y, añado, de la pasión por el derecho solo es posible si podemos mostrarles los dos aspectos de la experiencia jurídica que he mencionado antes y que solo la reflexión filosófico-jurídica y la teoría del derecho están en capacidad de iluminar. El primer aspecto es la naturaleza artificial del derecho: el derecho es construido por nosotros, por la política y, ante todo, por la cultura jurídica, y proporciona a la política el lenguaje en el que vienen formulados los problemas sociales y sus soluciones. El segundo aspecto lo representa la enorme brecha entre la normatividad fuerte y exigente de los principios de justicia establecidos en nuestras cartas constitucionales - la igualdad, la dignidad de las personas y sus derechos fundamentales- y el incremento de facto de las desigualdades y de las violaciones de los derechos producto del desarrollo desregulado de los poderes salvajes, tanto al interior de nuestros ordenamientos, cuanto $-\mathrm{y}$ aún más- en el mundo globalizado de hoy. Estos dos aspectos de la fenomenología del derecho exigen de la cultura jurídica, y sobre todo de la filosofía y teoría del derecho, un doble punto de vista crítico respecto del derecho vigente: la crítica externa, desde el punto de vista de la la justicia, y la crítica interna, desde el punto de vista de la legitimidad constitucional.

\section{EL SIGNIFICADO DEL POSITIVISMO JURÍDICO Y EL PUNTO DE VISTA CRÍTICO EXTERNO}

El primer punto de vista crítico es el generado por el primero de los dos aspectos mencionados anteriormente: la total artificialidad del derecho y de las instituciones jurídicas y políticas. Todo el derecho, todas las normas jurídicas, todas nuestras instituciones - las constituciones, las leyes y las prácticas judiciales, administrativas y negociales - son el producto de las elecciones de los hombres, resultantes de sus valores e intereses. Y todos nosotros, empezando por nosotros los juristas, tenemos - por como el derecho y las instituciones funcionan en concreto y, ante todo, por como son producidas, diseñadas y concebidas— responsabilidad en ello. 
Éste es el significado profundo del positivismo jurídico. Con el positivismo jurídico y con la afirmación del principio de legalidad como norma de reconocimiento del derecho existente nació la política moderna en cuanto producción y transformación, precisamente, del derecho, es decir, de las reglas y de las relaciones sociales. El lenguaje del derecho ha devenido, desde entonces, el lenguaje de la política, en el que, repito, vienen formulados los problemas sociales y sus soluciones. Esto conlleva dos corolarios.

El primer corolario del principio de legalidad es la separación entre derecho y justicia y la autonomía del derecho positivo vigente respecto del punto de vista de la moral y la política, y viceversa. Si damos por verdadero que es derecho todo y solo lo que es producido por el legislador, entonces su existencia y su validez se separan de la justicia, en el sentido de que no todas las normas jurídicas son justas solo porque sean válidamente producidas y jurídicamente existentes, y a la inversa, no todas las normas existen como normas jurídicas solo porque sean justas. El significado de la tan discutida «separación entre derecho y moral» no consiste más que en eso. Con dicha tesis no se quiere decir, por supuesto, que en el derecho no haya contenidos morales (o inmorales), sino únicamente que ni la existencia ni la validez jurídica de una norma implican la justicia de aquella, ni que la justicia de una norma implica la existencia y la validez jurídica de la misma. Por efecto del principio de legalidad se produce, en suma, una virtual divergencia entre el «deber ser moral o político» del derecho y su «ser» efectivo, es decir, entre el derecho existente y el derecho justo. De esto se sigue la refutación de una doble falacia ideológica: la del legalismo ético, que identifica la justicia con el derecho, y la del moralismo jurídico que identifica el derecho con la justicia. Esta virtual separación entre derecho y justicia es la que da lugar al campo de acción propio del punto de vista moral y político, autónomo y externo al derecho positivo: este punto de vista ético-político, señaladamente, el de la justicia, es crítico respecto del derecho existente cuando este es considerado injusto, y es proyectivo de un derecho más justo que el existente; es a partir de ese punto de vista —añado, no objetivo, sino subjetivo de cualquiera de nosotros- que se formulan las políticas de gobierno pero también las políticas de la oposición, la defensa del derecho existente pero también su crítica, y la proyección del derecho futuro.

El segundo corolario, conectado con el primero, es el rol pragmático del que están investidas tanto la política como la teoría del derecho y la filosofía de la justicia. Si el derecho es producto de la política, entonces, antes que nada, él ha sido pensado y teorizado por los hombres y, por tanto, por la filosofía jurídica y política. Debido a esto, bien podríamos decir que los padres constituyentes del moderno Estado de derecho han sido, más que los hombres políticos, los filósofos de la política y del derecho, de HobBes a Locke y a MontesQuieu, de BeCCARIA y FilangIERI a Kant y a CONSTANT. De aquí la dimensión pragmática y la responsabilidad de la filosofía jurídica y política en la teorización y en el diseño del derecho y de las instituciones políticas. La democracia, en efecto, es una construcción jurídica, así como una construcción política y social, y como tal supone un diseño teórico que solo la filosofía política y la teoría del derecho están en capacidad de desarrollar. 


\section{EL SIGNIFICADO DEL CONSTITUCIONALISMO JURÍDICO Y EL PUNTO DE VISTA CRÍTICO INTERNO}

El segundo punto de vista crítico en el examen del derecho vigente, que, a mi juicio, una aproximación filosófico-jurídica a la altura de nuestras democracias constitucionales sugiere al jurista, es el generado por el segundo de los dos aspectos de la moderna fenomenología del derecho que he mencionado en un inicio: la artificialidad, pero no solo del derecho, sino también de los principios y valores a los cuales el derecho debe ajustarse, y en consecuencia, la distancia que separa a tales principios de la realidad efectiva del derecho vigente. Gracias a la introducción de las constituciones rígidas, en efecto, todo el derecho, para ser válido y también existente, debe, por un lado, ser compatible y, por otro, actuar* los principios de justicia -la igualdad, la dignidad de la persona, los derechos fundamentales, tanto de libertad como sociales - estipulados en aquellas normas de derecho supraordenadas a todas las demás que son las formuladas en las cartas constitucionales, sean nacionales o internacionales.

Este es el significado y la sustancia del constitucionalismo jurídico, por lo que bien podríamos caracterizarlo como un positivismo jurídico reforzado y completado, al haber ampliado las opciones, que de otro modo serían arbitrarias, a las que debe ajustarse la legislación. Las constituciones diseñan, en efecto, el proyecto jurídico del futuro: los principios de libertad, de igualdad y de justicia que las leyes ordinarias deben garantizar y actuar y que equivalen a otros tantos límites y vínculos impuestos a la legislación y por tanto a la política. La política sigue siendo la fuente y el motor de la producción jurídica. Mas, al mismo tiempo, ella se subordina al derecho expresado en las constituciones, es decir, a la prohibición de lesionar los derechos de libertad y a la obligación de actuar los derechos sociales constitucionalmente establecidos, los cuales imponen dar, el uno un paso atrás, y el otro, un paso delante en la esfera pública. De aquí se siguen, de nuevo, otros dos corolarios.

El primer corolario del principio de legalidad constitucional consiste en una segunda separación: la separación no solo entre justicia y validez, sino también entre validez y existencia del derecho. Si es verdad que deviene existente todo y solo el derecho producido en las formas por él mismo establecidas, entonces, la existencia (o el vigor) de las normas se separa también de su validez jurídica, así como de su justicia: bien es posible, en efecto, que cobren existencia normas incompatibles con los principios constitucionales, y sean, por tanto, inválidas, y que no cobren existencia todas las normas de actuación implicadas e impuestas por los principios constitucionales. Bien es posible, en otras palabras, que se produzca un derecho ilegítimo, o sea, el ejercicio ilegítimo del poder, por comisión o por acción: una figura — la del derecho ilegítimoque el viejo positivismo jurídico — piénsese solamente en Hans KELSEN- concebía

N. del T.: El término italiano attuazione, que Luigi FERRAJOLI usa en varios pasajes del texto, forma parte de su lenguaje teórico axiomatizado. Lo hemos traducido como actuación, siguiendo la versión castellana de Principia iuris (Trotta, 2011). Así, cuando FerRAJOli nos hable del derecho, de las leyes o de las normas de actuación, hemos de entender que se refiere a la producción legislativa de las garantías primarias de los derechos sociales (es decir, de las obligaciones positivas correlativas a estos, cuya ausencia origina una laguna). 
como una «contradicción en los términos» y que constituye el rasgo característico de las actuales democracias constitucionales: su posible defecto, pero también su mayor mérito. solo en los ordenamientos en los cuales la potestad legislativa es absoluta, existencia y validez efectivamente coinciden. A causa de la virtual divergencia entre «deber ser» constitucional y «ser» legislativo del derecho, en cambio, en los ordenamientos dotados de constituciones rígidas existen, virtual pero inevitablemente, antinomias y lagunas.

El segundo corolario es el ulterior rol pragmático del que está investida la cultura jurídica, por efecto de la ampliación del punto de vista crítico, que incluye no solamente el político o externo, sino también el jurídico o interno al derecho positivo: este es, precisamente, el punto de vista de los principios constitucionales, crítico respecto del derecho existente cuando este sea inválido, y proyectivo de las normas de actuación implicadas e impuestas por dichos principios cuando ellas no existan. Gracias a este segundo punto de vista, es directamente la ciencia jurídica, mas no la filosofía política o de la justicia, la que está comprometida, en el plano científico antes que en el civil y político, con el rol crítico y proyectivo respecto de su propio objeto. De ahí surge el que bien podríamos llamar carácter «militante» de la ciencia jurídica, impuesto por las constituciones tomadas en serio: por un lado, la crítica de las normas inválidas, es decir, las antinomias, para que estas sean anuladas por la jurisdicción; por otro lado, el diseño de las leyes de actuación de los derechos establecidos, para que sus lagunas sean removidas por la legislación.

En este cambio del rol de la ciencia jurídica se manifiesta el nexo isomórfico existente entre derecho y sistema político, y en particular, entre las propiedades estructurales de los actuales ordenamientos jurídicos y la democracia. Esta relación consiste en la correspondencia entre las condiciones, tanto formales como sustanciales, de la validez de las normas jurídicas y las condiciones, formales y sustanciales, de la legitimidad del sistema político. En los Estados absolutos, quod principi placui legis habet vigorem et validitatem, resulta válido todo lo que agrada al soberano, cuya voluntad es la fuente de legitimación de todo el sistema político. En la democracia política del Estado legislativo, la validez de las normas está determinada por las formas político-representativas de la producción legislativa, correspondientes únicamente a la dimensión formal, política o representativa de la democracia. En la democracia constitucional, en fin, la legitimidad jurídica está determinada, además de por la conformidad de las normas producidas con las formas político-representativas de su producción, también por sus contenidos, en cuanto a la actuación y al respeto de los principios de justicia constitucionalmente establecidos, los que corresponden, a su vez, a la dimensión sustancial de la democracia constitucional.

\section{LA INSIDIA DEL REALISMO Y LOS PROBLEMAS DE LA ANOMIA}

Finalmente, en mi opinión, hay una última toma de conciencia que debe ser promovida por la aproximación filosófico-jurídica a nuestros ordenamientos de democracia constitucional. Me refiero a la insidia continua proveniente de las falacias del realismo jurídico y político. Evidentemente, la investigación empírica y sociológica sobre 
el funcionamiento concreto de nuestros ordenamientos es el presupuesto básico de cualquier aproximación científica al derecho. Sin embargo, conviene evitar una doble falacia.

La primera falacia es común a todas las ciencias sociales - a la ciencia jurídica, pero también a la teoría política y a la economía- y consiste en su concepción como ciencia puramente «descriptiva» y en la caracterización de su objeto como un objeto natural: como si el derecho, las instituciones jurídicas y políticas, el mismo funcionamiento del mercado fueran, de hecho, fenómenos naturales y no fenómenos artificiales producidos por la política, respecto de los cuales, entonces, la política lleva responsabilidad. «No hay alternativas», es la tesis que expresa esta naturalización, por ende privada de alternativas, de los fenómenos sociales, y que viene siendo repetida en estos años por nuestros gobernantes y por gran parte de los economistas para sostener las políticas de austeridad que están llevando a Europa a la ruina.

La segunda falacia está ligada específicamente a la naturaleza normativa del derecho y, en particular, a la normatividad fuerte de las normas constitucionales. Esta consiste en calificar como válido el derecho efectivamente practicado y, por tanto, en ignorar y, de hecho, secundar el eventual derecho ilegítimo, es decir, las violaciones, por comisión o por omisión, de las normas a él supraordenadas. En ambos casos se produce una especie de legitimación cruzada: de la teoría por parte de la realidad o sea de lo que acontece y de la realidad es decir de lo que acontece y de las prácticas jurídicas extra-legales por parte de la teoría; de la tesis teórica de la falta de alternativas por parte de la verificación de facto del derecho existente y de las ordenaciones económicas y sociales de las cuales ese derecho es expresión, e inversamente, del derecho existente aunque sea en contraste con su deber ser constitucional por parte de su descripción realista como privado de alternativas.

Pues bien, es precisamente la alternativa de las promesas constitucionales la que resulta desacreditada como «irrealista» por la tesis «realista» de la falta de alternativas. El distinguir entre el «deber ser» (jurídico) y el «ser efectivo» del derecho, así como entre la aproximación teórica, crítica y proyectiva, y la aproximación sociológica al derecho existente, es la condición necesaria, por tanto, para identificar el derecho ilegítimo y para dar cuenta, en el plano científico, de la complejidad estructural de nuestros ordenamientos.

En nuestro Congreso, una sesión específica ha estado dedicada a las varias formas de anomia. Hay pues, dos tipos de anomia — ambas ilegítimas, pero legitimadas, o en todo caso ignoradas, por las aproximaciones realistas- que, en mi opinión, representan hoy los principales problemas, no solamente políticos, sino también sociales. En primer lugar, el problema de las lagunas de garantías de los derechos sociales, que es grave, sobre todo, en los países de América latina. Todos los derechos fundamentales, si se los toma en serio, requieren leyes de actuación que introduzcan sus garantías, es decir, las correspondientes obligaciones a cargo de la esfera pública, sin las cuales están destinados a permaneces inefectivos: la institución del servicio sanitario y gratuito como garantía del derecho a la salud, la legislación sobre la escuela —igualmentepública y gratuita como garantía del derecho a la educación, la renta mínima de ciudadanía y las otras formas de previsión como garantía del derecho a la subsistencia. No 
se trata de opciones políticas confiadas a la discrecionalidad de los gobiernos, sino de deberes jurídicos de actuación, correlativos a esas específicas expectativas positivas de prestaciones públicas en las que consisten todos los derechos sociales. Por lo demás, contrariamente al lugar común neoliberal según el cual tales derechos son realistamente actuables en la medida en que existan los recursos económicos necesarios para actuarlos, nuestra experiencia enseña que los gastos sociales para satisfacerlos han sido las inversiones más productivas, incluso en el plano económico: la prueba de esto es la mayor riqueza de los países europeos respecto del resto del mundo y de su mismo pasado, debida al hecho de que ellos han garantizado - con la salud, la educación y los mínimos vitales - la máxima productividad individual y, por tanto, la máxima productividad colectiva.

La segunda anomia, aún más grave y dramática, es la ausencia de reglas heterónomas que estén a la altura de los poderes económicos y financieros globales. En el momento en el que el derecho estatal parecía haber alcanzado su máximo perfeccionamiento, gracias a los límites y vínculos impuestos a todos los poderes por los derechos fundamentales constitucionalmente establecidos, los poderes económicos y financieros se han reubicado fuera de los confines nacionales, evadiéndose del rol de gobierno de la política y de las funciones de garantía del derecho. Esta es, hoy en día, la verdadera amenaza para la democracia, tanto en su dimensión política y representativa como en su dimensión legal y constitucional: el incremento incontrolado de los poderes salvajes del mercado, producto de la asimetría entre el carácter global de la economía y las finanzas, y el carácter todavía preeminentemente estatal de la política y del derecho. Gracias a esta asimetría, se ha invertido la jerarquía democrática de los poderes: no es más la política la que gobierna la economía y las finanzas, sino a la inversa; no son más los representantes parlamentarios los que dirigen la política de los gobiernos y dictan reglas a los poderes económicos y financieros, sino que son estos poderes los que dictan sus reglas a los gobiernos y a los parlamentos, hasta el punto de exigir reformas constitucionales idóneas para asegurar la máxima gobernabilidad, es decir, la omnipotencia de la política respecto de la sociedad, necesaria para su impotencia y subordinación a los dictados de la economía y de las finanzas.

La globalización de la economía y de las finanzas, pero no de la política ni mucho menos del derecho y de los derechos, consiste precisamente en esta pérdida de soberanía política estatal, sustituida por el nacimiento de este nuevo soberano global, invisible, anónimo, irresponsable y al mismo tiempo voraz y depredador, constituido por los poderes desregulados del mercado. Consiste, por tanto, en el vacío de un derecho público global, colmado por el derecho privado producido por los mismos actores de la economía y de las finanzas; en la consecuente sustitución del imperio de la ley y su heteronomía por el imperio del contrato y de la autonomía privada como fuente privilegiada de la producción jurídica; en suma, en la afirmación como verdadera grund-norm del orden mundial, no ya de las constituciones nacionales y las cartas internacionales de los derechos, sino de la ley del mercado.

Me parece que repensar la geografía de poderes, tanto públicos como privados, y reivindicar el rol del derecho como ley del más débil en contra de las leyes de los más fuertes, que son las leyes de la economía, son actualmente las tareas principales de 
la filosofía del derecho del futuro. Se trata de una batalla cultural, en la que, quizás, la cultura jurídica y la filosofía del derecho del Mundo Latino estén mejor provistas que la cultura y la filosofía jurídica anglosajonas, más ligadas a la tradición tanto del realismo jurídico como del neoliberalismo económico. Esto bastaría para justificar nuestros encuentros y nuestro compromiso de cara a una discusión y un intercambio futuros. 